\title{
MODELIZACIÓN ESPACIAL DE LAS POBLACIONES DE Oligonychụs perseae (TUTTLE, BAKER Y ABATIELLO, 1976) EN EL ESTADO DE MÉXICO
}

\section{SPATIAL MODELING OF THE Oligonychus perseae (TUTTLE, BAKER AND ABATIELLO, 1976) POPULATIONS IN THE STATE OF MEXICO}

\author{
Ana V. Lara-Diaz, José F. Ramírez-Dávila*, Fatima I. Maldonado-Zamora, \\ Roberto Rivera-Martínez, Agustín D. Acosta-Guadarrama y Fidel Lara-Vázquez
}

Universidad Autónoma del Estado de México, Facultad de Ciencias Agrícolas, Toluca, Estado de México, México.

*Autor de correspondencia (jframirezd@uaemex.mx)

\section{RESUMEN}

Oligonychus perseae es un ácaro conocido como araña cristalina que afecta al cultivo de aguacate (Persea americana), prácticamente a nivel mundial. En países como Estados Unidos de América y España se reporta como la principal plaga que afecta el follaje del aguacatero. Los daños incluyen afectación de los tejidos celulares de las hojas debido a la alimentación del ácaro, defoliación y hasta daños por mancha de sol debido a la baja cantidad de follaje en el árbol. El umbral económico de esta plaga se reporta entre 100 y 500 ácaros por hoja. La finalidad del presente estudio fue modelizar el comportamiento de las poblaciones de araña cristalina en cuatro municipios productores de aguacate en el Estado de México. Los resultados mostraron que los municipios más afectados por esta plaga fueron Donato Guerra y Temascaltepec. Se observó que el estrato más afectado fue el bajo con 40 arañas por hoja, en el que se encontraron hasta 130 ácaros por árbol. Las poblaciones de araña cristalina se distribuyen de manera agregada en los municipios muestreados y se apegan a modelos esféricos y gaussianos principalmente. Todos los muestreos se validaron adecuadamente mediante el proceso de validación cruzada. La distribución de las poblaciones se ilustró mediante mapas de densidad que muestran la ubicación precisa de los centros de agregación en los municipios muestreados. Con base en los mapas se calculó la superficie infestada, la que supera el $57 \%$ de la superficie de los municipios evaluados.

Palabras clave: Olygonichus perseae, México, modelización espacial.

\section{SUMMARY}

Oligonychus perseae is a mite known as persea mite that affects avocado (Persea americana) plantations practically worldwide. It is reported as the main pest affecting avocado tree foliage in countries like the United States of America and Spain. Damages include injuries to the cell tissues of the leaves due to mite feeding, defoliation and even sunspot damages due to the low amount of foliage on the tree. The economic threshold of this pest is reported between 100 and 500 mites per leaf. The purpose of this study was to model the behavior of persea mite populations in four avocadoproducing municipalities in the State of Mexico, Mexico. Results showed that the municipalities most affected by this pest were Donato Guerra and Temascaltepec. It was observed that the most affected stratum was the lower one with $\mathbf{4 0}$ mites per leaf, in which up to 130 mites per tree were found. Persea mite populations are distributed in an aggregate way in the sampled municipalities and mainly correspond to spherical and Gaussian models. All samplings were properly validated through the cross-validation process. The distribution of populations was illustrated by density maps that show the precise location of aggregation centers in the sampled municipalities. Based on the maps, the infested area was calculated, it exceeds $57 \%$ of the area of the evaluated municipalities.

Index words: Olygonichus perseae, Mexico, spatial modeling.

\section{INTRODUCCIÓN}

Oligonychus perseae es un ácaro ampliamente distribuido a nivel mundial, y en México ha sido reportado en todas las plantaciones de aguacate (Persea americana) en altas densidades, con excepción de las ubicadas en climas templados húmedos, donde las incidencias son bajas (Coria y Ayala, 2010). Actualmente, su distribución se ha ampliado conforme se incrementan las zonas aguacateras. Según Aponte y McMurtry (1997), este ácaro se alimenta del follaje y provoca manchas necróticas de color rojizo en las hojas debido a la inserción de los estiletes en los tejidos de la planta y en ataques muy severos provoca defoliación (Ochoa et al., 1994). De acuerdo con Alcázar et al. (2005, Com. Pers.) ${ }^{1}$, el daño que origina el ataque de ácaros en aguacate puede reducir hasta en 30 \% la actividad fotosintética de las hojas. Hoddle (2008) reportó que la susceptibilidad de los cultivares es variable, el cultivar Fuerte resultó el menos susceptible al ataque del ácaro seguido de Lamb Hass, Reed, Esther, Pinkerton, Gwen y Hass, este último fue el más susceptible, pues presentó poco más del $38 \%$ de la superficie de la hoja con daños por la presencia de araña cristalina.

Se han realizado diversos trabajos para controlar esta plaga, uno de ellos es la investigación de Bouriga-Valdivia et al. (2016) en Zirimícuaro y Ziracuaretiro, Michoacán, mediante la aplicación del insecticida orgánico agrosiamil ${ }^{\circledR}$

'Alcázar M. D., G. Aranda, A. L. Márquez, L. Sánchez and C. Ruiz (2005) Oligonychus perseae (Acari: Tetranychidae). Una nueva plaga en el aguacate en el sur de España. In: IV Congreso Nacional de Entomología Aplicada. Braganza, Portugal. p:213. 
acompañado del adherente ovicida gardytec $^{\circledast}$ donde encontraron efectividad en las dosis de 2 y $3 \mathrm{~mL} \mathrm{~L}^{-1}$ de agua, solo y acompañado con $2 \mathrm{~mL} \mathrm{~L}^{-1}$ de adherente. SalvadorDe Jesús et al. (2016) con control biológico, reportaron como principal depredador natural de ácaros en el cultivo de aguacate a Euseius hibisci Chant, este ácaro pertenece a la familia Phytoseiidae y está ampliamente distribuido; por su parte, Flores et al. (2011) señalaron que gracias a la capacidad depredadora de E. hibisci se reducen las poblaciones de 0 . perseae a niveles que no representan importancia. El control de los ácaros en aguacatero se realiza en mayor medida mediante la aplicación de abamectina, azufre mojable, azadiractin y jabón potásico en sus diferentes presentaciones comerciales, todo lo anterior con las implicaciones ecológicas que genera en la entomofauna benéfica, ya que estos productos, pese a ser orgánicos, originan daños en el sistema nervioso de una gran variedad de insectos.

La determinación de los posibles patrones de distribución a los que se ajusta una población de insectos o ácaros en un cultivo dado ayuda a conocer sus hábitos de vida, su tendencia a agruparse en las diferentes etapas de desarrollo y las preferencias de hábitat (Southwood, 1995). Una alternativa para describir los patrones de distribución espacial es el uso de la geoestadística, que emplea información acerca del valor que toma la variable y además de la ubicación espacial de los puntos de muestreo a fin de resumir la correlación entre puntos. Dado lo anterior, y ante los costos ecológicos que genera la aplicación de agroquímicos y otros compuestos usados en la agronomía, la aplicación de la geoestadística en la agricultura surge como una alternativa para minimizar el impacto que genera la producción de alimentos y reducir la cantidad de insumos aplicados, ya que esta técnica favorece la aplicación dirigida a lugares donde se encuentran poblaciones en altas densidades dejando libres las zonas donde las poblaciones son mínimas o nulas. El estudio tuvo por objeto modelizar el comportamiento de 0 . perseae mediante la aplicación de técnicas geoestadísticas e ilustrar su distribución en cuatro municipios productores de aguacate en el Estado de México, mediante mapas obtenidos por medio del krigeado ordinario.

\section{MATERIALES Y MÉTODOS}

\section{Área de estudio}

El estudio se realizó en cuatro municipios del Estado de México (Coatepec Harinas, Temascaltepec, Donato Guerra y Tenancingo), donde la importancia en la producción de aguacate es destacada. Coatepec Harinas se encuentra entre los paralelos $18^{\circ} 48^{\prime}$ y $19^{\circ} 05^{\prime} \mathrm{N}$, los meridianos $99^{\circ}$ $43^{\prime} y 9^{\circ} 54^{\prime}$ O, con altitud entre 1600 y 3900 m. Donato
Guerra se ubica en las coordenadas $19^{\circ} 24^{\prime} \mathrm{N}, 100^{\circ} 19^{\prime}$ $\mathrm{O}$, a una altitud media de $2200 \mathrm{msnm}$. Temascaltepec se ubica al sur del Estado a una altitud media de 1720 msnm en las coordenadas $19^{\circ} 02^{\prime} \mathrm{N}$ y $100^{\circ} 02^{\prime} \mathrm{O}$, mientras que Tenancingo tiene su ubicación a los $19^{\circ} 59^{\prime} \mathrm{N}$ y $99^{\circ} 10^{\prime} \mathrm{O}$ con una altitud media de 2020 msnm.

\section{Muestreo}

Se muestrearon 200 árboles distribuidos y marcados a lo largo y ancho de los municipios evaluados mediante el método de cuadrantes. Los árboles muestreados se seleccionaron en plantaciones de 10 años de edad. Se efectuaron conteos quincenales de los adultos de O. perseae. Se seleccionaron 60 hojas del estrato bajo, medio y alto del árbol tomando de referencia los puntos cardinales del árbol. Cada árbol fue georreferenciado con un navegador GARMIN modelo eTrex Vista HCx2007 (Olathe, Kansas, EUA), de acuerdo con los ensayos hechos por Lara-Velázquez et al. (2018). Para las observaciones se usó una lupa de aumento de 20X, los muestreos se realizaron del 1 de julio al 22 de diciembre de 2017.

\section{Análisis de la información}

Una vez obtenidos los datos en campo, se realizó la estimación de los semivariogramas experimentales; el semivariograma se calculó mediante la fórmula propuesta por Journel y Huijbregts (1978) e Isaaks y Srivastava (1989).

$$
Y *(h)=\frac{1}{2 N(h)} \sum_{(i=1)}^{N(H)}\left[z\left(x_{i}+h\right)-z\left(x_{i}\right)\right]^{2}
$$

Cualquier función matemática se puede utilizar para un modelo de semivariograma, siempre que sea positiva y definida (Armstrong y Jabin, 1981). Para el semivariograma experimental correspondiente a cada muestreo se empleó el programa Variowin 2.2 (software para el análisis de datos espaciales en 2D. Primavara Verlag, New York, EUA).

Los modelos teóricos, comúnmente, usados para ajustar los semivariogramas experimentales son: esférico, exponencial, gaussiano, logarítmico, efecto pepita puro, efecto agujero y monómico (Samper y Carrera, 1996; Trematerra y Sciarretta, 2002). Los parámetros del modelo a validar ( $\mathrm{C}_{0}$, efecto pepita, $\mathrm{C}$, meseta y a, rango o alcance) se van modificando hasta la obtención de estadísticos de validación cruzada adecuados, los cuales son:

1. Media de los errores de estimación (MEE)

2. Error cuadrático medio (ECM)

3. Error cuadrático medio adimensional (ECMA) (Hevesi et al., 1992). 
Se calculó el nivel de dependencia espacial con el fin de determinar la robustez de la relación entre los datos del muestreo. Este valor se obtiene al dividir el efecto pepita entre la meseta, expresado en porcentaje: menos de $25 \%$ se considera alto, entre 26 y $75 \%$ es moderado y superior al 76 \% se considera bajo (Cambardella et al., 1994; LópezGranados et al., 2002).

Se elaboraron mapas mediante la interpolación de valores a través del krigeado ordinario, que permite la estimación no sesgada de valores asociados a puntos que no fueron muestreados, todo esto con base en lo establecido por Samper y Carrera (1996). Finalmente, se estableció la superficie infestada de las estimaciones y se representaron en forma de mapas para cada municipio por medio del programa Surfer 10 (Surface Mapping System, Golden Software Inc. Golden, Colorado, USA).

\section{RESULTADOS Y DISCUSIÓN}

Se generó la modelización espacial de las poblaciones de o. perseae con la aplicación de métodos geoestadísticos y se determinó el patrón de la distribución del ácaro en los cuatro municipios muestreados. La distribución espacial de araña cristalina se presentó en agregados dentro de los municipios evaluados. Otros métodos usados para determinar la agregación de plagas agrícolas han sido reportados en los trabajos de Silva et al. (2016) y Vivas y Notz (2011). Se determinó la dependencia espacial que existe entre los agregados encontrados en los huertos de producción, esto debido a la concentración de ácaros por hoja encontrados en cada muestreo. El estrato más afectado corresponde a la parte baja del árbol, ya que fue donde se encontró la mayor cantidad de ácaros por hoja (40), y se manifestaron hasta 130 ácaros por árbol.

El mayor número de individuos por árbol se encontró durante los meses de julio, agosto y septiembre, aunque hubo una disminución en este último debido a la reducción de las temperaturas, lo que limitó su reproducción, al limitar la actividad diaria y la tasa de reproducción de los ácaros. Aunque en los meses de mayor precipitación ocurre una disminución abrupta de las poblaciones de ácaros tetraníquidos como 0 . punicae a causa de la lluvia como un factor supresor de las poblaciones de ácaros, O. perseae persiste en condiciones de Iluvia moderada (Sánchez et al., 2001).

El comportamiento de araña cristalina en aguacate se apegó, principalmente, al modelo gaussiano en Coatepec Harinas (Cuadro 1) y a modelos esféricos en los municipios de Donato Guerra (Cuadro 2), Temascaltepec (Cuadro 3) y Tenancingo (Cuadro 4). Estos modelos explican ampliamente la disposición espacial de las plagas al mostrar que las poblaciones se presentan en centros de agregación dentro de las parcelas, y en el caso del modelo esférico muestra una disposición espacial aleatoria de los centros de agregación de la plaga, mientras que el modelo gaussiano implica una distribución continua dentro de las áreas de muestreo. Ambos modelos han explicado correctamente el comportamiento de otras plagas, un ejemplo de ello lo presenta Jiménez et al. (2013), donde el modelo gaussiano es el que mejor explica la distribución espacial de huevos de trips en tomate de cáscara (Physalis ixocarpa), mientras que en tomate (Solanum lycopersicum), los modelos que mejor explican la distribución de la

Cuadro 1. Parámetros de efecto pepita, rango, meseta, dependencia espacial y superficie infestada de los modelos ajustados a los semivariogramas de Oligonychus perseae en aguacate en Coatepec Harinas, Estado de México.

\begin{tabular}{|c|c|c|c|c|c|c|}
\hline Modelo & Pepita & Rango & Meseta & Pepita /Meseta & Dependencia espacial & Superficie infestada (\%) \\
\hline Esférico & 0 & 15.23 & 61.2 & 0 & Alta & 100 \\
\hline Exponencial & 0 & 23.26 & 65.99 & 0 & Alta & 100 \\
\hline Exponencial & 0 & 18.7 & 26.51 & 0 & Alta & 100 \\
\hline Exponencial & 0 & 24.77 & 49.10 & 0 & Alta & 100 \\
\hline Gaussiano & 0 & 14.3 & 74.99 & 0 & Alta & 99 \\
\hline Gaussiano & 0 & 14.63 & 54.94 & 0 & Alta & 100 \\
\hline Gaussiano & 0 & 17.6 & 49.45 & 0 & Alta & 100 \\
\hline Esférico & 0 & 12.1 & 28.05 & 0 & Alta & 99 \\
\hline Gaussiano & 0 & 19.03 & 20.68 & 0 & Alta & 97 \\
\hline Gaussiano & 0 & 16.5 & 11.25 & 0 & Alta & 99 \\
\hline Esférico & 0 & 12.1 & 8.40 & 0 & Alta & 100 \\
\hline Esférico & 0 & 18.13 & 1.96 & 0 & Alta & 100 \\
\hline
\end{tabular}


Cuadro 2. Parámetros de efecto pepita, rango, meseta, dependencia espacial y superficie infestada de los modelos ajustados a los semivariogramas de Oligonychus perseae en aguacate en Donato Guerra, Estado de México.

\begin{tabular}{lcccccc}
\hline Modelo & Pepita & Rango & Meseta & Pepita /Meseta & Dependencia espacial & Superficie infestada (\%) \\
\hline Esférico & 0 & 13.2 & 39.25 & 0 & Alta & 100 \\
Gaussiano & 0 & 11.50 & 13.85 & 0 & Alta & 85 \\
Esférico & 0 & 16.5 & 68.04 & 0 & Alta & 100 \\
Gaussiano & 0 & 17.6 & 10.33 & 0 & Alta & 99 \\
Esférico & 0 & 20.9 & 79.15 & 0 & Alta & 100 \\
Gaussiano & 0 & 16.5 & 50.66 & 0 & Alta & 100 \\
Esférico & 0 & 21.49 & 82.43 & 0 & Alta & 97 \\
Esférico & 0 & 12.1 & 60.84 & 0 & Alta & 96 \\
Gaussiano & 0 & 22.0 & 10.81 & 0 & Alta & 98 \\
Esférico & 0 & 16.92 & 11.48 & 0 & Alta & 98 \\
Esférico & 0 & 16.9 & 10.264 & 0 & Alta & 96 \\
Esférico & 0 & 19.37 & 10.90 & 0 & Alta & 97 \\
\hline
\end{tabular}

Cuadro 3. Parámetros de efecto pepita, rango, meseta, dependencia espacial y superficie infestada de los modelos teóricos ajustados a los semivariogramas de Oligonychus perseae en aguacate en Temascaltepec, Estado de México.

\begin{tabular}{lcccccc}
\hline Modelo & Pepita & Rango & Meseta & Pepita /Meseta & Dependencia espacial & Superficie infestada (\%) \\
\hline Esférico & 0 & 21.49 & 26.02 & 0 & Alta & 100 \\
Esférico & 0 & 18.53 & 12.29 & 0 & Alta & 100 \\
Gaussiano & 0 & 14.63 & 71.49 & 0 & Alta & 99 \\
Esférico & 0 & 19.71 & 61.22 & 0 & Alta & 60 \\
Gaussiano & 0 & 16.5 & 88.18 & 0 & Alta & 57 \\
Gaussiano & 0 & 11.0 & 72.70 & 0 & Alta & 88 \\
Esférico & 0 & 12.1 & 45.78 & 0 & Alta & 98 \\
Esférico & 0 & 15.06 & 9.07 & 0 & Alta & 99 \\
Esférico & 0 & 12.1 & 11.69 & 0 & Alta & 100 \\
Esférico & 0 & 17.6 & 8.87 & 0 & Alta & 91 \\
Gaussiano & 0 & 11.0 & 8.15 & 0 & Alta & 99 \\
Esférico & 0 & 19.44 & 2.17 & 0 & Alta & 98 \\
\hline
\end{tabular}

plaga Helicoverpa armigera Hübner son el esférico y el exponencial (Moral et al., 2004) debido a que las plagas se distribuyen erráticamente dentro de distancias muy cortas en pequeñas parcelas de cultivo (Isaaks y Srivastava, 1989), pero dada la naturaleza del presente estudio, las distancias entre los puntos de muestreo y la poca movilidad de o. perseae, tanto el modelo esférico como el gaussiano explican el comportamiento de los ácaros.
El valor del efecto pepita fue cero para todos los muestreos realizados en los cuatro municipios, se observó que el 100 \% de la variación de la distribución de 0 . perseae se explica por la estructura espacial establecida en los semivariogramas respectivos (Cuadros 1 a 4), lo cual indica un reducido error de muestreo; este valor es relevante debido al comportamiento errático de las plagas dentro de las parcelas e influye en el proceso posterior a la estimación (Moral, 2004). El valor del efecto pepita indicó 
Cuadro 4. Parámetros (efecto pepita, rango, meseta, dependencia espacial y superficie infestada) de los modelos teóricos ajustados a los semivariogramas de Oligonychus perseae en aguacate en Tenancingo, Estado de México.

\begin{tabular}{lcccccc}
\hline Modelo & Pepita & Rango & Meseta & Pepita /Meseta & Dependencia espacial & Superficie infestada (\%) \\
\hline Esférico & 0 & 15.4 & 31.15 & 0 & Alta & 100 \\
Gaussiano & 0 & 11.25 & 75.68 & 0 & Alta & 99 \\
Gaussiano & 0 & 10.40 & 25.70 & 0 & Alta & 97 \\
Esférico & 0 & 12.86 & 24.92 & 0 & Alta & 100 \\
Esférico & 0 & 13.2 & 25.94 & 0 & Alta & 73 \\
Esférico & 0 & 15.65 & 68.05 & 0 & Alta & 99 \\
Esférico & 0 & 13.2 & 45.04 & 0 & Alta & 99 \\
Gaussiano & 0 & 13.4 & 32.17 & 0 & Alta & 93 \\
Esférico & 0 & 13.36 & 15.57 & 0 & Alta & 99 \\
Esférico & 0 & 16.83 & 15.36 & 0 & Alta & 99 \\
Esférico & 0 & 15.56 & 8.54 & 0 & Alta & 99 \\
Esférico & 0 & 15.65 & 8.50 & 0 & Alta & 100 \\
\hline
\end{tabular}

que la distancia entre los puntos muestrales fue adecuada, ya que a medida que se alejaron los puntos de captura fue más notable el efecto pepita. Los valores dentro del intervalo apropiado de los estadísticos de la validación cruzada permitieron validar los modelos ajustados para cada muestreo (Cuadros 1 a 4).

Los valores del rango pertenecientes al municipio de Coatepec Harinas oscilaron entre 12.1 y 24.77 m (Cuadro 1 ), en el municipio de Donato Guerra los valores oscilaron entre 11.50 y 22.0 m (Cuadro 2), en Temascaltepec se obtuvieron valores de entre 11.0 y 21.49 m (Cuadro 3) y la variación en Tenancingo fue de 10.40 a 16.83 m (Cuadro 4). Esta información es importante, ya que refleja la distancia máxima hasta la que existe dependencia espacial entre los datos obtenidos durante los muestreos. Este mismo análisis fue realizado por Ramírez et al. (2011) para determinar la distancia para la que existe dependencia espacial en Bactericera cockerelli Sulc en el cultivo de papa (Solanum tuberosum).

Por su parte, Márquez-Pérez et al. (2018) determinaron el patrón espacial de Huanglongbing (HLB) en limón persa (Citrus $\times$ latifolia) y corroboraron una distribución espacial agregada y una dependencia espacial a $40 \mathrm{~m}$ de distancia con la participación del psílido asiático de los cítricos Diaphorina citri Kuwayama como vector reportado en México, lo que afectó hasta el $95 \%$ de las plantaciones de limón. Con los resultados obtenidos del valor del efecto pepita y el valor de la meseta se calculó la dependencia espacial cuyo valor en todos los casos fue alto (Cuadros 1 a 4$)$.

El cálculo de la dependencia espacial ha sido usado en múltiples investigaciones, una ellas es el estudio sobre la distribución espacial de Aedes aegypti Linneaus en Puerto Carreño, Colombia, donde se encontró una dependencia espacial media en el comportamiento del vector de dengue y un ajuste al modelo esférico (Niño, 2011).

Los porcentajes de la superficie infestada indican que en la mayoría de los muestreos en los cuatro municipios llegó al $100 \%$ de los árboles muestreados, como se observa en los Cuadros 1 a 4. La menor superficie infestada estuvo en el muestreo del mes de septiembre en el municipio de Temascaltepec con una superficie infestada del $57 \%$. La información se puede visualizar mejor a través de los mapas en los que se observa la agregación de los ácaros, donde los patrones de agregación son indicativos de la presencia de interacciones entre los ácaros y el medio, o entre los mismos organismos (Figuras 1 a 4); se pueden observar áreas libres e infestadas por 0 . perseae; si bien esta especie se presenta en los meses más calurosos, en los muestreos realizados se encontró un comportamiento irregular, debido a la impermeabilidad de las telarañas las colonias no se vieron afectadas por las lluvias y los ácaros permanecieron protegidos y esta condición no mermó las poblaciones del ácaro perseae. Comportamientos iguales fueron observados por Equihua et al. (2007), quienes mencionaron que los ácaros, para protegerse de la lluvia y de las altas humedades, construyen un mayor número de colonias protegidas por telarañas y esto provoca aumento de las poblaciones al inicio de las lluvias.

Las técnicas geoestadísticas aplicadas en el control de plagas han permitido tener conocimiento de la distribución espacial, lo que contribuye a conocer sus hábitos de vida y se ve expresado en los modelos de dispersión en las zonas 

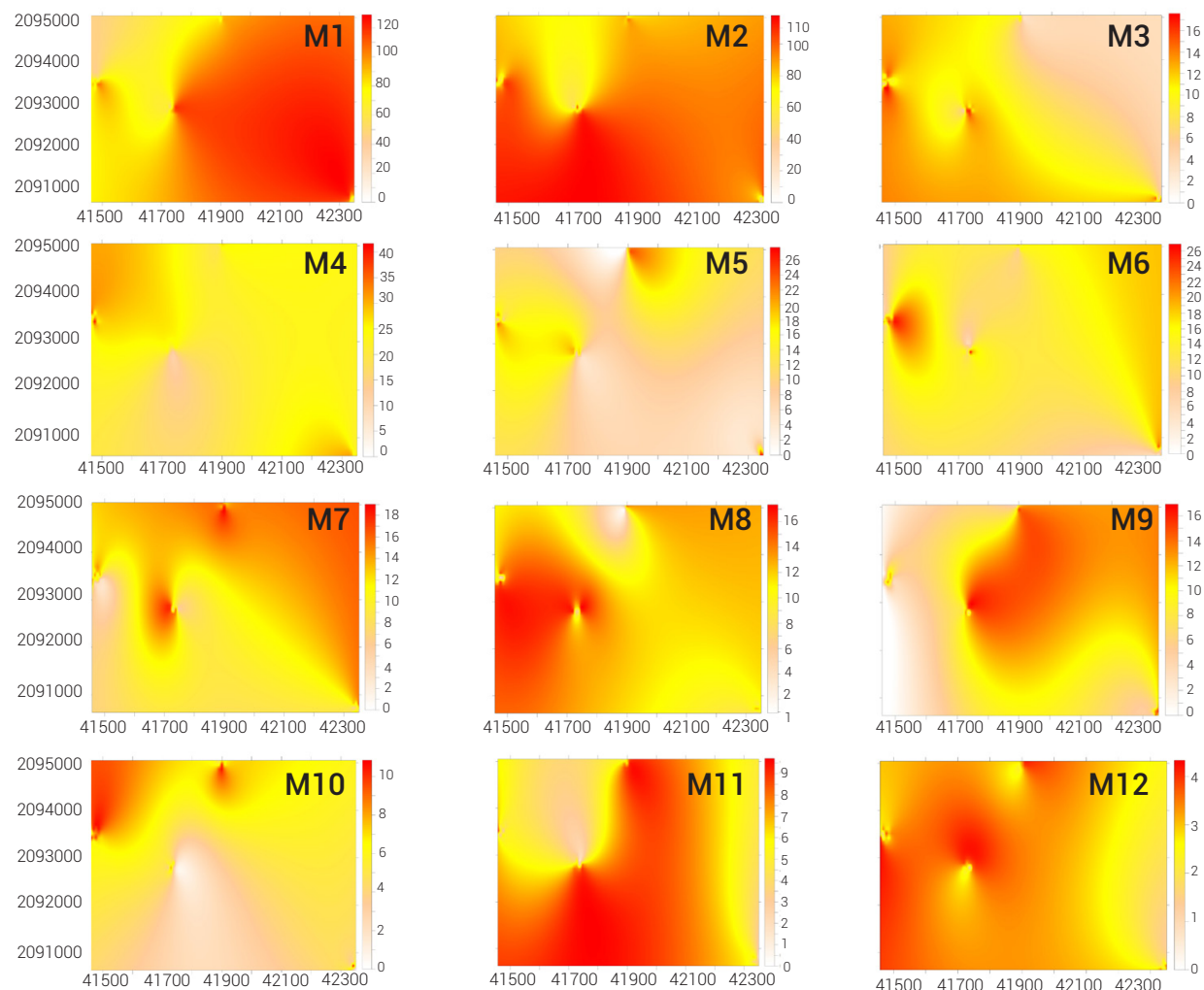

Figura 1. Mapas de densidad de las poblaciones de Oligonychus perseae en el cultivo de aguacate en Coatepec Harinas, Estado de México.
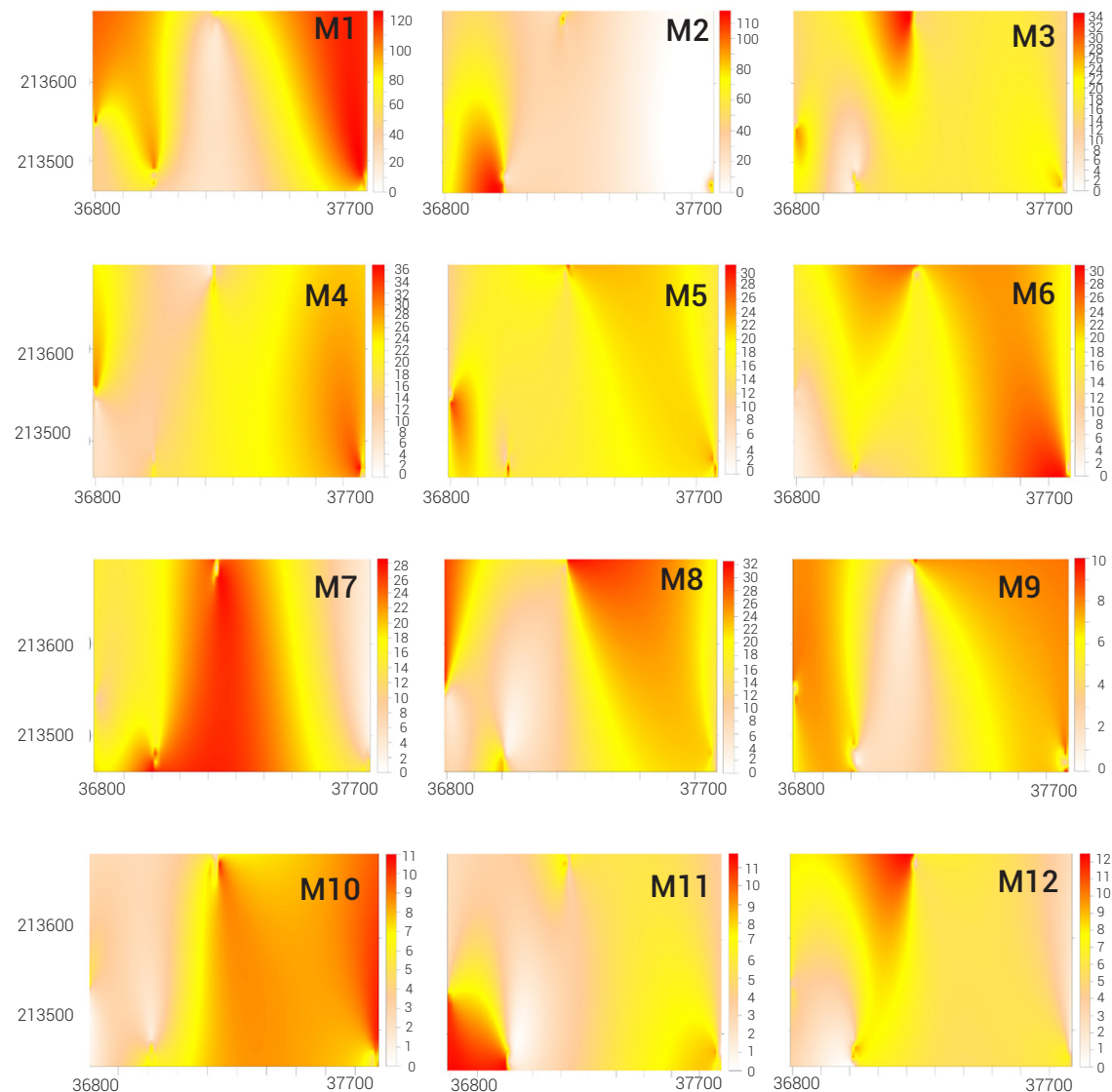

Figura 2. Mapas de densidad de las poblacıones de Olıgonychus perseae en el cultıvo de aguacate en Donato Guerra, Estado de México. 

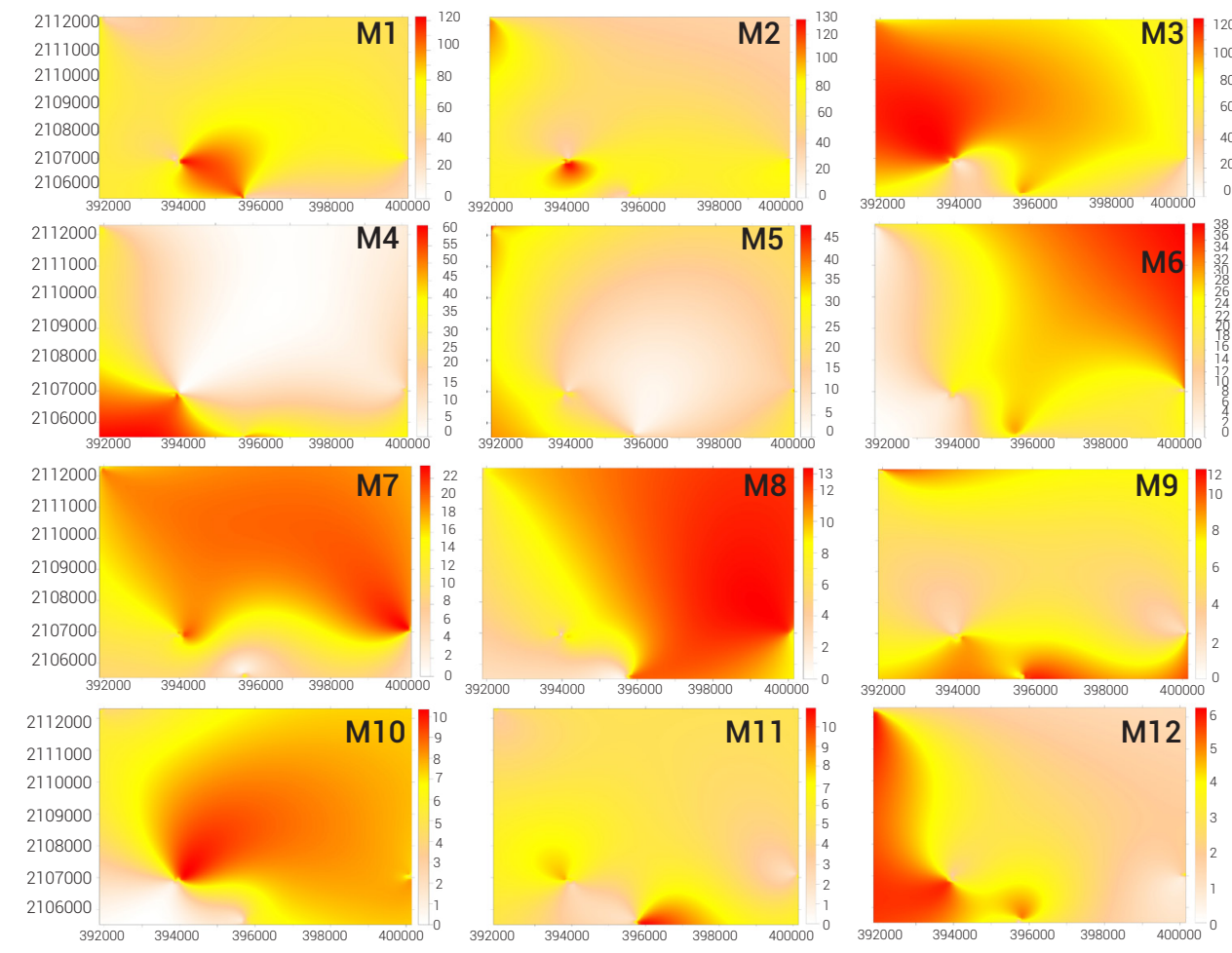

Figura 3. Mapas de densidad de las poblaciones de Oligonychus perseae en el cultivo de aguacate en Temascaltepec, Estado de México.
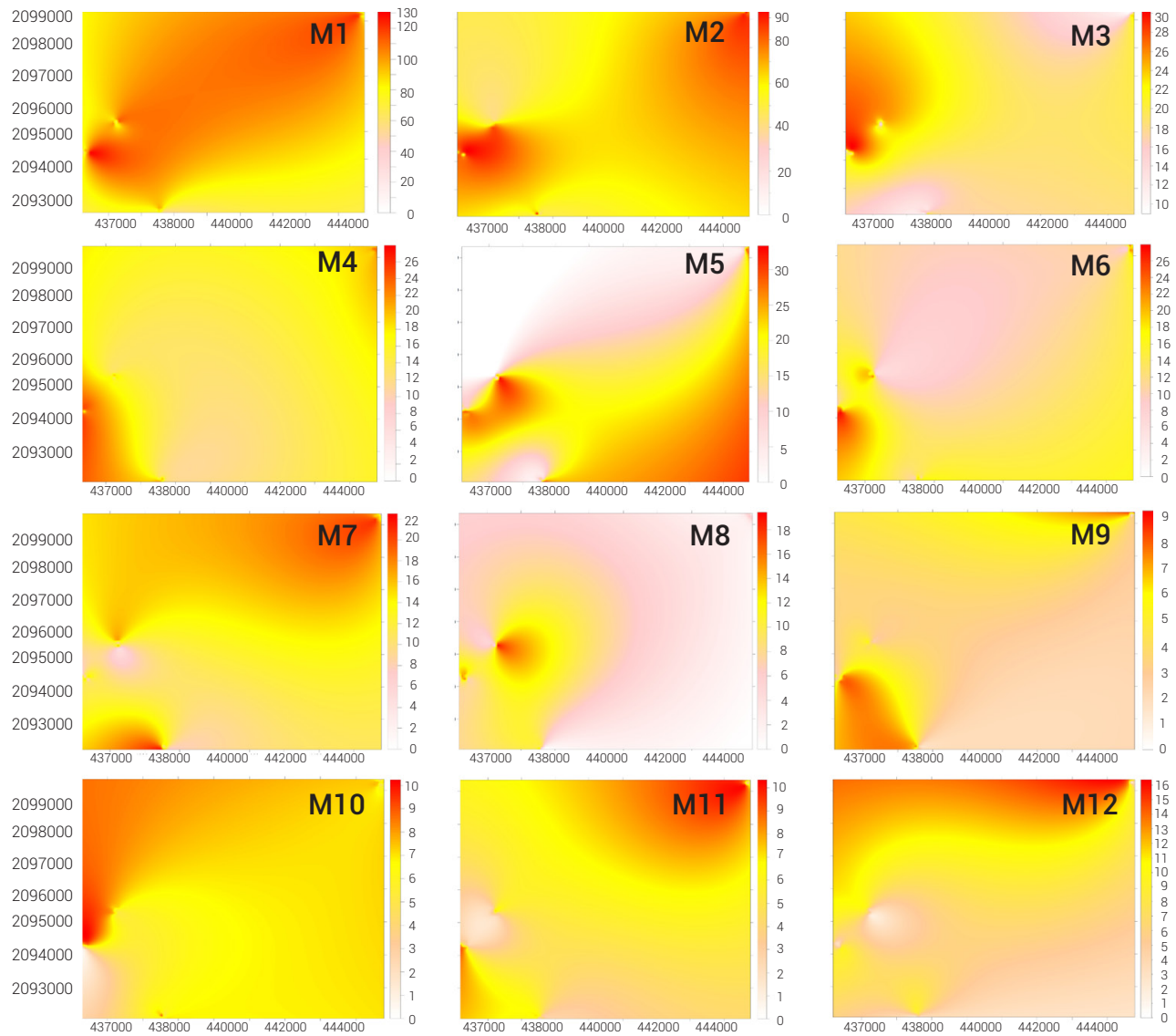

Figura 4. Mapas de densidad de las poblaciones de Oligonychus perseae en el cultivo de aguacate en Tenancingo, Estado de México. 
de muestreo. La información obtenida permite crear planes de control en diferentes etapas de desarrollo del ácaro, y de esta manera implementar programas de manejo integrado de plagas dentro de las zonas de cultivo de aguacate. La ilustración de la presencia de araña cristalina del aguacatero se realizó mediante el proceso de krigeado ordinario; este método de estimación geoestadística consiste en proporcionar el valor más probable de una variable espacial en un punto; el krigeado es un estimador ELIO (estimador lineal, insesgado y óptimo) que posee diversos métodos propuestos para abordar situaciones tanto teóricas como prácticas (Chica-Olmo, 2005).

Los mapas generados para los cuatro municipios ilustran la presencia de los centros de agregación con el mayor número de ácaros (color rojo), y que a medida que disminuye la presencia de estos organismos la intensidad del color disminuye a amarillo, y en ausencia de arañas se torna blanco (Figuras 1 a 4). Los mapas permitieron determinar los focos de infestación de 0 . perseae y con ello hacer un uso racional de los acaricidas para proteger la fauna entomológica benéfica, además de predecir las zonas donde se desarrollarán poblaciones altas en las siguientes temporadas, en función de los niveles de población al final de la anterior. Estos mapas contribuyen a decidir dónde y cuándo aplicar diversas medidas de control, puesto que la recurrente presencia del ácaro en las parcelas de cultivo de aguacate en el Estado de México ha provocado que los productores apliquen métodos de control químico a lo largo del año.

Evidencias directas de los productores de las zonas evaluadas indican que el control de ácaros en las plantaciones de aguacate se lleva a cabo con aplicación de abamectinas, que tienen un costo de alrededor de $\$$ $1,000.00$ pesos mexicanos por hectárea, lo que representa una alta inversión en las huertas. Para el presente estudio, la aplicación de acaricidas se focalizó en las áreas con coloración roja de los mapas (Muestreos 1, 2, 11 y 12) en el municipio de Coatepec Harinas (Figura 1), Muestreo 7 en Donato Guerra (Figura 2), Muestreos 7 y 8 en el municipio de Temascaltepec (Figura 3) y Muestreo 1 en Tenancingo (Figura 4), pero éstas se podrían reducir o evitar si no superan los umbrales económicos y al considerar que en muchas ocasiones las aplicaciones no son realizadas de manera correcta y algunos organismos han generado resistencia a piretroides y organofosforados; sin embargo, Ramos-Gutiérrez et al. (2015) mencionan que 0 . perseae no ha mostrado resistencia según el estudio de susceptibilidad a acaricidas realizado en huertos de aguacate en Nayarit, México.

En los mapas las áreas en blanco y amarillo son indicativas de superficies donde se puede aplicar un control alternativo como el control biológico; puesto que los niveles de ácaros son inferiores a 10 individuos por árbol es recomendable el uso de ácaros depredadores como los de la familia Phytoseiidae. Kerguelen y Hoddle (1999) demostraron que Neoseiulus californicus McGregor y Galendromus helveolus Chant son las especies con mayor eficiencia en reducir las poblaciones de este ácaro en plantaciones de aguacate en California y que estos depredadores controlan mejor la plaga cuando ésta se encuentra en bajas densidades. También se podría optar por contar con fauna endémica auxiliar en el control de 0 . perseae, pero para ello se requiere eliminar la aplicación de agroquímicos que afecten las poblaciones de estos organismos.

\section{CONCLUSIONES}

El uso de técnicas geoestadísticas representa una alternativa para el control de plagas de ácaros. La mayor cantidad de individuos de Oligonychus persea se encontró en el estrato bajo del árbol con hasta 40 ácaros por hoja. Los municipios más afectados por esta plaga son Temascaltepec y Donato Guerra en el Estado de México. El modelo que representa mejor el comportamiento espacial de 0 . persea es el modelo esférico. 0 . persea presenta rangos promedio de movilidad de $16.5 \mathrm{~m}$ y puede infestar hasta $100 \%$ de los huertos de aguacate en el Estado de México. La ilustración de los modelos de dispersión de los ácaros permite orientar las medidas de control a las zonas específicas donde se encuentra la mayor cantidad de organismos plaga y en las fechas donde se incrementan las poblaciones de ácaros.

\section{BIBLIOGRAFÍA}

Aponte 0. and J. A McMurtry (1997) Biology, life table and mating behavior of Oligonychus perseae (Acari: Tetranychidae) International Journal of Acarology 23:199-207, https://doi. org/10.1080/01647959708683564

Armstrong M. and R. Jabin (1981) Variogram models must be positive-definite. Journal of the International Association for Mathematical Geology 13:455-459, https://doi.org/10.1007/ BF01079648

Bouriga-Valdivia E., M. Vargas-Sandoval, J. J. Ayala-Ortega, M. B. N. LaraChávez y M. Contreras-Gutiérrez (2016) Evaluación de insecticidas orgánicos para el control de ácaros en el cultivo del aguacate. Entomología Mexicana 3:125-130.

Cambardella C. A., T. B. Moorman, J. M. Novak, T. B. Parkin, D. L. Karlen, R. F. Turco and A. E. Konopka (1994) Field scale variability of soil properties in central lowa soils. Soil Science Society of America Journal 58:1501-1511, https://doi.org/10.2136/ sssaj1994.03615995005800050033x

Chica-Olmo M. (2005) La geoestadística como herramienta de análisis espacial de datos de inventario forestal. Cuadernos de la Sociedad Española de Ciencias Forestales 19:47-55.

Coria A. V. M. y A. Ayala S. (2010) Manejo de ácaros del aguacate en México. Folleto Técnico Núm. 20. Campos Experimentales Uruapan y Zacatepec, INIFAP. Uruapan, Michoacán, México. 19 p.

Equihua M. A., V. E. G. Estrada y H. H. González (2007) Plagas del aguacate. In: El Aguacate y su Manejo Integrado. D. Téliz O. y A. Mora 
(coords.). Segunda edición. Mundi-Prensa. México, D.F. pp:135-169.

Flores C. R. J., N. Isiordia A., A. Robles B., O. Ortega Á., R. Pérez G. y A. Ramos Q. (2011) Ácaros fitófagos asociados a frutales en la zona de Nayarit. Revista Fuente 2:25-33.

Hevesi J. A., J. D. Istok and A. L. Flint (1992) Precipitation estimation in mountainous terrain using multivariate geostatistics. Part I. Structural analysis. Journal of Applied Meteorology and Climatology 31:661-676, https://doi.org/10.1175/15200450(1992)031<0661:PEIMTU>2.0.C0;2

Hoddle M. (2008) Plagas del palto en California, México y Centroamérica In: Manejo de Plagas en Paltos y Cítricos. R. Ripa y P. Larral (eds.). Instituto de Investigaciones Agropecuarias. Santiago, Chile. pp:317-323

Isaaks E. H. and R. M. Srivastava (1989) An Introduction to Applied Geostatistics. Oxford University Press. New York, USA. 561 p.

Jiménez C. R. Á., J. F. Ramírez D., J. R. Sánchez P., M. L. Salgado S. y A. Laguna C. (2013) Modelización espacial de Frankliniella occidentalis (Thysanoptera: Thripidae) en tomate de cáscara por medio de técnicas geoestadísticas. Revista Colombiana de Entomología 39:183-192.

Journel A. G. and C. J. Huijbregts (1978) Mining Geostatistics. Academic Press. London, England. $600 \mathrm{p}$.

Kerguelen V. and M. Hoddle (1999) Biological control of Oligonychus perseae (Acari: Tetranychidae) on avocado: II. evaluating the efficacy of Galendromus helveolus and Neoseiulus californicus (Acari: Phytoseiidae). International Journal of Acarology 25:221-229, https://doi.org/10.1080/01647959908684156

Lara-Velázquez F., J. F. Ramírez-Dávila, M. Rubí-Arriaga, E. J. Morales-Rosales, D. K. Figueroa-Figueroa, A. D. Acosta-Guadarrama and R. RiveraMartínez (2018) Distribución espacial de araña roja Oligonychus punicae Hirst en el cultivo del aguacate, en dos municipios del Estado de México. Southwestern Entomologyst. 43:743-759, https://doi.org/10.3958/059.043.0320

López-Granados F., M. Jurado-Expósito, S. Atenciano, A. García-Ferrer, M. Sánchez 0. and L. García-Torres (2002) Spatial variability of agricultural soil parameters in southern Spain. Plant and Soil 246:97-105, https://doi.org/10.1023/A:1021568415380

Márquez-Pérez F. J., J. L. Flores-Sánchez, L. Rodríquez-Mejía, J. MárquezGómez, S. J. Michereff, V. Ancona, ... and S. Domínguez-Monge (2018) Progress and spatial pattern of huanglongbing in Persian lime in Nayarit, México. Revista Bio Ciencias 5:e351, https://doi. org/10.15741/revbio.05.02.01

Moral G. F. J. (2004) Aplicación de la geoestadística en las ciencias ambientales. Ecosistemas 13:78-86.

Moral G. F. J., J. A. Rodríguez B., A. Arranz R., J. J. de la Cruz B. y F. Honorio G. (2004) Técnicas geoestadísticas aplicadas al análisis de la distribución de capturas de Helicoverpa armigera (Hübner)
(Lepidoptera: Noctuidae) mediante trampas con feromonas sexuales en una plantación de tomate. Boletín de Sanidad Vegetal de Plagas 30:733-744.

Niño L. (2011) Interpolación espacial de la abundancia larval de Aedes aegypti para localizar focos de infestación. Revista Panamericana de Salud Pública 29:416-422

Ochoa R., H. Aguilar and C. Vargas (1994) Phytophagous Mites of Central America: An Illustrated Guide. CATIE. Turrialba, Costa Rica. 234 p.

Ramírez D. J. F., E Porcayo C. y J. R. Sánchez P. (2011) Análisis de la distribución espacial de Bactericera cockerelli Sulc (Hemiptera: Triozidae) en Solanum tuberosum L. en Donato Guerra, México. Boletín del Museo de Entomología de la Universidad del Valle 12:12-24.

Ramos-Gutiérrez F. A., C. Santillán-Ortega, A. Robles-Bermúdez, N. IsiordiaAquino, M. García-López, R. J. Flores-Canales and M. S. GonzálezCorona (2015) Susceptibility to acaricides in Oligonychus perseae from avocado orchards in Nayarit, México. Revista Bio Ciencias 3:220-227, https://doi.org/10.15741/revbio.03.03.08

Salvador-De Jesús L. A., E. G. Estrada-Venegas, A. Equihua-Martínez y M. P. Chaires-Grijalva (2016) Relación Oligonychus perseae (Prostigmata: Tetranychidae) y Euseius hibisci (Mesostigmata: Phytoseiidae) en dos huertas de aguacate en Uruapan, Michoacán. Entomología Mexicana 3:115-119.

Samper C. F. J. y J. Carrera R. (1996) Geoestadística: Aplicaciones a la Hidrología Subterránea. Segunda edición. Centro Internacional de Métodos Numéricos en Ingeniería. Barcelona. 484 p.

Sánchez P. J. L., J. J. Alcántar R., V. M. Coria A., J. Anguiano C., I. Vidales F., L. M. Tapia V., ... y J. A. Vidales F. (2001) Tecnología para la Producción de Aguacate en México. Libro Técnico No. 1. INIFAP, Campo Experimental Uruapan. Uruapan, Michoacán. 208 p.

Silva A. S., T. A. Mota, N. G. Piñeyro, M. G. Fernandes y F. F. Pereira (2016) Distribución espacial de Vatiga spp. (Hemiptera: Tingidae) en el cultivo de yuca. Acta Biológica Colombiana 21:195-200 https://doi.org/10.15446/abc.v21n1.46762

Southwood T. R. E. (1995) Ecological Methods. With Particular Reference to the Study of Insects Population. Second edition. Chapman \& Hall. New York, USA. 524 p.

Trematerra P. and A. Sciarretta (2002) Phenology and spatial analysis of some Coleoptera infesting a feed mill. In: Proceedings of the 8th International Working Conference on Stored-Product Protection. P. F. Credland, D. M. Armitage, C. H. Bell, P. M. Cogan, E. Highley (eds.). 22-26 July 2002. CAB International. York, UK pp:276-280.

Vivas C. L. E y A. Notz (2011) Distribución espacial en poblaciones de Oebalus insularis Stal (Hemiptera: Pentatomidae) en el cultivo de arroz en Calabozo, estado Guárico, Venezuela. Revista Científica UDO Agrícola 11:109-125. 
